\title{
Interpretación Conforme: Tendencias en su Aplicación por Tribunales Mexicanos. ${ }^{1}$
}

\author{
Conforming Interpretation: Trends in its Application by Mexican Courts.
}

\author{
Lucila Caballero Gutiérrez. ${ }^{2}$
}

Sumario: Introducción. II. El Estado de Derecho y la fundamentación de decisiones de órganos públicos. III. La decisión de interpretación y su justificación. IV. La interpretación conforme. V. Tendencias de decisiones judiciales en torno a la interpretación conforme. VI. Conclusiones.

\section{Resumen}

Actualmente una de las tendencias en interpretación jurídica por parte de tribunales mexicanos, sobre todo en derechos humanos, es la interpretación conforme. Ello derivado de la reforma del 10 de junio de 2011 al artículo primero constitucional. La reforma busca maximizar la protección de derechos humanos contenidos en diversos ordenamientos $\mathrm{y}$ en tratados internacionales. En este trabajo tenemos por objetivo general indagar y reflexionar acerca del deber de "interpretación conforme", para lo cual hemos de contestar las siguientes preguntas: ¿tal deber de interpretación conforme existía o no previamente a la adición al artículo primero constitucional en la reforma del 2011?En caso afirmativo hemos de responder a la pregunta ¿cuáles son las tendencias actuales en este tipo de interpretación conforme, a partir de la reforma del 2011? Para alcanzar nuestro objetivo general y responder las preguntas planteadas haremos el análisis de algunos casos resueltos por tribunales mexicanos, en especial, a partir de criterios jurisprudenciales. Dada la naturaleza de nuestro objeto de estudio, la metodología será básicamente hermenéutica, tomando como punto de partida el discurso jurídico plasmado en documentos oficiales publicados por la Suprema Corte de Justicia de la Federación, por lo que la técnica será de tipo documental. La aportación que propone este trabajo, es hacer visible la dirección que ha tomado

\footnotetext{
${ }^{1}$ Ponencia presentada en el I Congreso Nacional y VIII Coloquio tendencias Actuales del Derecho

${ }^{2}$ Profesora investigadora de la División de Ciencias Sociales de la Universidad de Sonora integrante del CAC Retos Actuales de las Ciencias Jurídicas y Sociales
} 
recientemente la interpretación jurídica conforme, en la actuación de los principales intérpretes del derecho en un país, los jueces.

Palabras clave: Interpretación conforme, derechos humanos, tribunales mexicanos

\begin{abstract}
One of the trends in legal interpretation by Mexican courts, especially in human rights, is currently the conforming interpretation. This branch of the reform of June 10 of 2011 to the first article constitutional. The reform seeks to maximize the protection of human rights contained in various legal codes and international treaties. In this work we have general objective investigate and reflect on the duty of "conforming interpretation", to which we have to answer the following questions: such duty of conforming interpretation or not existed prior to the addition to the article first constitutional reform of the 2011 ? If so, we have to answer the question what are the current trends in this type of conforming interpretation, since the reform of the 2011? To achieve our overall objective and answer the questions we will do analysis of some cases resolved by Mexican courts, in particular from case law criteria. Given the nature of our object of study, the methodology will be basically hermeneutics, taking as starting point the legal discourse reflected in official documents published by the Supreme Court of Justice of the Federation, what technique will be documentary. The contribution that proposes this work, is make visible the direction that has taken recently it conforming interpretation as, in the resolutions of the main interpreters of the law in a country, the judges.
\end{abstract}

Key words: conforming interpretation, human rights, Mexican courts

\title{
Introducción
}

Actualmente una de las tendencias en interpretación jurídica por parte de tribunales mexicanos, sobre todo en derechos humanos, es la interpretación conforme. Ello derivado de la reforma del 10 de junio de 2011 al artículo primero constitucional. Reforma que busca maximizar la protección de derechos humanos contenidos en diversos ordenamientos y tratados internacionales. 
En este trabajo tenemos por objetivo general indagar y reflexionar acerca del deber de "interpretación conforme", para lo cual hemos de contestar las siguientes preguntas: ¿tal deber de interpretación conforme existía o no previamente a la adición al artículo primero constitucional en la reforma del 2011 ?

En caso afirmativo hemos de responder a la pregunta ¿cuáles son las tendencias actuales en este tipo de interpretación conforme, a partir de la reforma del 2011 ?

Para alcanzar nuestro objetivo general y responder las preguntas planteadas haremos el análisis de algunos casos resueltos por tribunales mexicanos, en especial, a partir de criterios jurisprudenciales. Dada la naturaleza de nuestro objeto de estudio, la metodología será básicamente hermenéutica, tomando como punto de partida el discurso jurídico plasmado en documentos oficiales publicados por la Suprema Corte de Justicia de la Nación, por lo que la técnica será de tipo documental.

La aportación que propone este trabajo, es hacer visible la dirección que ha tomado recientemente la interpretación jurídica conforme, en la actuación de los principales intérpretes del derecho en un país, los jueces.

\section{El Estado de Derecho y la Fundamentación de Decisiones de Órganos}

\section{Públicos.}

En vista de que hemos de elaborar el análisis de algunos casos resueltos por tribunales mexicanos, en especial, a partir de criterios jurisprudenciales, partiremos de un concepto teórico básico para dicho análisis: el concepto de Estado de Derecho

De acuerdo al Artículo 16 constitucional, todo acto de autoridad ha de estar fundado y motivado. No cabe duda que este precepto constitucional es base fundamental de un Estado de derecho. Pero ¿cuáles son las características más importantes de un Estado de derecho? Gran cantidad de autores han escrito sobre la materia por lo que habremos de referenciar a algunos en la búsqueda de tales características.

A decir de Rodrigo Moreno, entre las características más importantes del Estado de Derecho pueden citarse: “a) toda la organización política de la comunidad se ajusta al régimen jurídico nacional; b) los particulares pueden inconformarse y atacar jurídicamente los actos de sus gobernantes a través de los recursos y procedimientos establecidos para el efecto; c) las potestades gubernamentales se encuentran definidas 
y delimitadas, y d) los derechos públicos subjetivos de los gobernados se encuentran garantizados por la Constitución. Pudiera decirse en síntesis que el Estado de derecho se caracteriza por el gobierno de las leyes y no de los hombres."3

En tanto que para Gustavo Zagrebelsky "El Estado de derecho indica un valor y alude sólo a una de las direcciones de desarrollo de la organización del Estado, pero no encierra en sí consecuencias precisas. El valor es la eliminación de la arbitrariedad en el ámbito de la actividad estatal que afecta a los ciudadanos. La dirección es la inversión de la relación entre poder y derecho que constituía la quintaesencia del Machtstaat (Estado bajo el régimen de fuerza) y del Polizeistaat (Estado bajo el régimen de policía): no más rex facit legem, sino lex facit regem" 4

Por su parte Manuel Atienza considera que "La idea del Estado de Derecho se vincula obviamente con la necesidad de que las decisiones de los órganos públicos estén argumentadas. Esto es así porque, en el contexto de un Estado de Derecho... la justificación de las decisiones no se hace depender sólo de la autoridad que las haya dictado, sino también del procedimiento seguido y del contenido. El Estado de Derecho ofrece, así, mayores posibilidades para la argumentación que cualquier otro tipo de argumentación del poder. En este sentido, cabría decir que la idea regulativa del estado de Derecho - si se quiere del Estado democrático de Derecho- es el sometimiento del Estado, del poder, a la razón y no de la razón al poder. Lo cual se conecta con la tendencia existente en las sociedades democráticas avanzadas -tendencia en la que no deja de haber excepciones - a otorgar una importancia creciente a la motivación -y a la justificación mediante argumentos- tanto en términos cuantitativos como cualitativos." 5

Como vemos en estas tres referencias de los autores al estado de derecho, la clave radica en el valor legal de una decisión estatal, hasta dónde y cómo debe de justificarse esta decisión. Decir que como gobernado debes hacer algo, ha de ser con base en razones y bajo cierto procedimiento o camino previamente establecido, sobre todo en una ley en donde se establezca el qué o contenido del deber, el quién o autoridad

\footnotetext{
${ }^{3}$ Moreno Rodríguez, Rodrigo, La administración pública federal en México, Universidad Nacional Autónoma de México, México, 1980, pp.32 y 33

${ }^{4}$ Zagrebelsky, Gustavo, El derecho dúctil. Ley derecho y justicia, ed. Trotta, Madrid, 2011, p.21.

${ }^{5}$ Atienza, Manuel, Cuestiones Judiciales, ed. Fontamara, colección BEFDP No 91, México, 2001, pp. 94 y 95.
} 
expresamente facultada para establecerlo, y el cómo o procedimiento necesario para hacerlo cumplir.

El modelo actual de estado de derecho no hubiese sido posible sin las ideas aportadas por tres grandes pensadores: Montesquieu, John Locke y Juan Jacobo Rousseau. El concepto de división de poderes y el principio de legalidad, sentaron las bases para un estado más equilibrado, y dieron pauta para el surgimiento de los derechos del hombre. Pasamos de un Estado absolutista en donde la voluntad del rey era la que prevalecía, como soberano absoluto, a un Estado en donde el poder es compartido y sujeto a diversos límites.

Teóricamente se ha clasificado al estado de derecho en dos aspectos: el formal y el material. Materialmente, el estado de derecho, aun cuando exige el respecto del principio de legalidad, tal legalidad ha de estar legitimada y ser acorde con los principios de justicia, fines y valores a los cuales debe servir el derecho. Esto si partimos de la idea de que el derecho es un medio, un instrumento, el cual, en consecuencia, y es lo más importante, necesario para alcanzar tales fines y principios.

Con el concepto formal de estado de derecho, se presenta la remisión al contenido del orden jurídico correspondiente. La garantía fundamental que se otorga consiste en que todos los actos provenientes del Estado deben ser actos fundados en el orden constitucional.

A partir de lo expuesto por Joseph Raz, podemos extraer algunos principios del estado de derecho (en especial un estado de derecho constitucional) tales como:

1. Todas las disposiciones jurídicas deben ser prospectivas, abiertas y claras.

2. Las disposiciones jurídicas deben ser relativamente estables.

3. El establecimiento de disposiciones jurídicas particulares (órdenes jurídicas particulares) debe ser guiado por disposiciones jurídicas abiertas, estables, claras y generales.

4. La independencia del Poder Judicial tiene que estar garantizada.

5. Los principios de justicia natural tienen que ser observados.

6. Los tribunales deben tener poderes de revisión sobre la implementación de otros principios.

7. Los tribunales deben ser fácilmente accesibles. 
8. A los órganos de prevención criminal dotados de discrecionalidad no se les debe permitir pervertir el derecho.

9. El establecimiento de disposiciones jurídicas particulares (órdenes jurídicas particulares) debe ser guiado por disposiciones jurídicas abiertas, estables, claras y generales.

10. La independencia del Poder Judicial tiene que estar garantizada.

11. Los principios de justicia natural tienen que ser observados.

12. Los tribunales deben tener poderes de revisión sobre la implementación de otros principios.

13. Los tribunales deben ser fácilmente accesibles.

14. A los órganos de prevención criminal dotados de discrecionalidad no se les debe permitir pervertir el derecho. ${ }^{6}$

Cabe aclarar que esta lista no es exhaustiva y que del concepto formal del derecho podemos, teóricamente, derivar otros principios; uno de ellos es el de creación y aplicación del derecho o, en otros términos, el principio de legalidad, conforme al cual las normas jurídicas de grada inferior deben ser creadas de acuerdo con las de grada superior. Así, todos los actos jurídicos se tienen que enmarcar en el sistema constitucional de que se trate.

No cabe duda que estos principios a los que alude Raz, son la base de la actuación de un estado racional y demócrata, pero no de cualquier democracia, o sea una de mera forma, sino de una democracia sustancial en la que hay auténtico respeto a los derechos humanos.

Para Luigi Ferrajoli, ${ }^{7}$ "la supervivencia de cualquier democracia necesita algún límite sustancial. Siempre es posible que con métodos democráticos se supriman los propios métodos democráticos y los derechos de libertad, sociales, políticos, el pluralismo político, la división de poderes, la representación... todo el sistema de reglas de la democracia política. Piénsese en las terribles experiencias totalitarias del

6 Véase Raz, Joseph, La autoridad del derecho. Ensayos sobre derecho y moral, Universidad Nacional Autónoma de México, México, 1985, pp. 278 y siguientes.

7 Ferrajoli, Luigi, Democracia constitucional y derechos fundamentales. La rigidez de la Constitución y sus garantías, http://www.fcje.org.es/wpcontent/uploads/file/jornada3/3_FERRAJOLlespa\%F1ol.pdf. 
fascismo y del nazismo, que conquistaron el poder de forma democrática y luego lo entregaron a un jefe que eliminó la democracia."

Ferrajoli $^{8}$ señala que la voluntad popular es auténtica sólo si puede expresarse libremente, y esto sucede sólo a través del ejercicio, además del derecho al voto, de las libertades fundamentales por parte de todos y cada uno. No existe soberanía popular sin derechos de libertad individual. La omnipotencia de la mayoría amenaza la democracia política y la propia soberanía popular, que sólo se realizan a través del ejercicio constante de los derechos de libertad. Estos últimos son efectivos si se sustentan en la garantía de derechos sociales a la subsistencia y a la salud y, aún más, en el derecho a la educación y a la información. Ferrajoli ${ }^{9}$ concluye que sin satisfacer estos derechos, tanto los políticos como los de libertad están destinados a permanecer en el papel. Sin la garantía de mínimos vitales: los derechos a la supervivencia a la educación y a la información, no existe participación en la vida pública ni formación de voluntad consciente. Es decir, para identificar un verdadero estado de derecho, estos mínimos vitales a los que alude Ferrajoli han de ser ejercidos fácticamente y las propias instituciones estatales han de actuar en torno a lograr su eficacia o en caso contrario, brindar todas las posibilidades para que se defiendan ante arbitrariedades cometidas y se subsanen los errores.

Conforme a lo anterior hemos de afirmar que el Estado de Derecho moderno, es un Estado Constitucional que se vincula con lo que Carbonell ${ }^{10}$ denomina "constitucionalización del sistema jurídico". Cabe aclarar una afirmación que el propio Carbonell señala, en el sentido de que no se puede aceptar cualquier contenido constitucional, sino un contenido que acepte "la ideología liberal democrática, comprometida con el respeto de los derechos fundamentales, con algunos valores mínimos que debe proteger inexorablemente" ${ }^{11}$ y agrega "es inadmisible que el texto constitucional tenga una "indiferencia valorativa e ideológica" como un recipiente vacío que puede establecer tanto una dictadura como una democracia."12

${ }^{8}$ Ferrajoli, Luigi, La teoría del derecho en el paradigma constitucional, México, Fontamara, 2010, pp.78 y 79

${ }^{9}$ Ferrajoli, Luigi, op. cit, pp. 80 y 81.

10 Carbonell, Miguel, Neo constitucionalismo y derechos fundamentales, Colombia, ed. Cevallos, 2011 pp. 31 a 36

${ }^{11}$ Carbonell, Miguel, Constitución, reforma constitucional y fuentes de derecho en México, 6a ed., México, Porrúa-UNAM, 2008, pp. 249 y 250

12 Idem 
Los anteriores conceptos de estado de derecho constitucional que respeta los derechos humanos, nos servirán de marco de referencia para identificar la tendencia en algunas decisiones de interpretación emitidas por tribunales mexicanos, en torno a la interpretación conforme. Antes de ello, hemos de partir de un concepto de interpretación jurídica y de directiva de interpretación, a fin sentar bases de nuestro marco de referencia.

\section{La decisión de interpretación y su justificación}

Por interpretación jurídica entendemos determinar o explicar el significado de normas de derecho, en donde cabe la posibilidad de más de un significado, derivado de los problemas propios del lenguaje natural por el que son dadas a conocer las normas: la vaguedad y la ambigüedad. El fin básico de una interpretación jurídica, sobre todo de las autoridades y en especial de los jueces, es, independientemente de la creencia en un obvio significado único o en la posibilidad de varios significados, fundar un acto de autoridad; es decir, en un Estado de Derecho que respete el principio moderno de la legalidad, significa decir a otros cual norma aplican y por qué los faculta a elegir cierto sentido de la norma aplicada.

Por otra parte, el ejercicio práctico de la interpretación jurídica llevado a cabo por diversos operadores jurídicos, conlleva el uso referencial de ciertas teorías del derecho y esto, sin duda, influye en la labor creativa del intérprete, en su posibilidad de ver el mundo de cierta manera y no de otra.

Hay otra razón por la que la labor de interpretación del derecho, especialmente la elaborada por los jueces, es una actividad creativa. Si partimos del supuesto de que interpretar jurídicamente implica reformular lingüísticamente el texto normativo interpretado a fin de regular el caso concreto. Lo anterior conlleva lo que Hans Kelsen denomina "establecer el sentido de la norma" ${ }^{13}$ actividad que necesariamente ha de realizar el órgano aplicador del derecho. El propio Kelsen ${ }^{14}$ llama la atención acerca de la indeterminación normativa, y plantea el problema del "marco de posibles significados normativos. Sin duda, este autor abrió la posibilidad a la discusión teórica acerca de los

\footnotetext{
${ }^{13}$ Kelsen, Hans, teoría Pura del Derecho, ed. Porrúa, México, 1997, p. 349.

${ }^{14}$ Véase, Kelsen, Hans, op. cit. pp.351 y 352
} 
problemas que conlleva esta posible indeterminación normativa. Algunos de estos problemas también surgen por ciertas antinomias o contradicciones entre normas. ${ }^{15}$

Guastini $^{16}$ alude a dos posibles razones por las que surgen los problemas de interpretación jurídica : a) los problemas que surgen por los defectos del propio lenguaje en que se formula el derecho y b) los problemas son ocasionados por las "construcciones dogmáticas de los intérpretes". En este último sentido consideramos que es valiosa la aportación de Guastini. Es valiosa porque deja ver que un teórico del derecho puede identificar problemas donde otra persona, carente de marco teórico conceptual, no ve. A esto le denomina Guastini "construcciones dogmáticas de los intérpretes."

Comunicarle a otro el sentido del derecho es necesario pero no suficiente al interpretar. Hace falta algo más: se requiere justificar o dar razones de esa explicación o sentido. En este punto se alude a directivas de interpretación, que en diferentes contextos reciben otras denominaciones, tales como métodos, técnicas, argumentos, entre otros. Entonces ¿cuál es, si la hay, la diferencia entre interpretación jurídica y directivas de interpretación jurídica? La diferencia radica en que aunque ambas se integran con enunciados, la primera sólo busca atribuir significados y la segunda, la directiva, busca justificar, probar el por qué de ése significado electo. A partir de esta afirmación nos preguntamos ¿existen algunas directivas o justificaciones que sean obligatorias para un juez? Nuestra respuesta es afirmativa, por lo que hemos de aludir a una de estas justificaciones o directivas jurídicas obligatorias: la conforme.

\section{La interpretación conforme}

Hay más de una forma de eliminar los conflictos normativos, sin embargo a partir de la reforma de junio de 2011 al artículo primero constitucional, se establece como obligación para toda autoridad, realizar una interpretación conforme, si se trata de normas relativas a los derechos humanos.

Según Esquiaga, la reforma en materia de derechos humanos en México se debió a una violación masiva de derechos derivada de la violencia terrorista, y afirma

${ }^{15}$ Véase al respecto, Nino Carlos S. Introducción al Análisis del Derecho, ed. Ariel, Barcelona, 1987 pp.272 a 275 2001, p. 58

${ }^{16}$ Guastini, Riccardo, Estudios sobre la Interpretación Jurídica, Porrúa, México, 
que tal reforma de la Constitución mexicana sólo contiene una directiva: la conforme. ${ }^{17}$ No compartimos la idea del autor, toda vez que consideramos que de menos se establecieron en la reforma dos directivas de interpretación muy importantes: la "conforme" y la "liberal restrictiva".

¿Tal deber de interpretación conforme existía o no previamente a la adición al artículo primero constitucional en la reforma del 2011?

Para darle sentido más amplio a la reforma del 2011 en cuanto a la interpretación conforme, tenemos que considerar que esta interpretación conforme se aplica también a las normas constitucionales, lo que implicaría su subordinación al derecho internacional. En caso contrario, la obligación de interpretación conforme ya estaría contemplada antes de la reforma en el artículo 133 de la constitución.

Cabe aclarar que antes de la reforma al primero constitucional, se denominaba "conforme", "adecuadora" o "de conformidad con la Constitución" a la justificación o directiva retórica de una interpretación, que tuviera por objeto interpretar cualquier normatividad diferente a la constitución y ver si era acorde o no con los preceptos constitucionales. Esta facultad se desprende aun actualmente, del artículo 133 constitucional, que dio pié a una gran cantidad de decisiones ya que comprende todo el contenido de la Constitución

Ahora, con base en la reforma del artículo primero constitucional, y por la "autoridad lingüística" que la Constitución representa, la directiva conforme se reduce al contenido que sobre derechos humanos tenga la Constitución y los tratados. Es de hacer notar que esto no excluye el uso de la antigua directiva retórica "conforme" del 133, sin embargo, ésta no se vincula con un derecho humano concreto sino con todos los derechos marcados en la Constitución.

Algunas posibles características de la interpretación conforme son:

- Constituye una técnica para determinar el contenido normativo de un orden jurídico en general, y de sus disposiciones jurídicas en particular;

17 Ezquiaga, Ganuzas, Francisco Javier, La interpretación de los derechos humanos de conformidad con la Constitución y con los tratados internacionales. El nuevo artículo $1^{\circ}$ de la Constitución mexicana, Revista del Instituto de la Judicatura federal, México, 2011 p. 191 
- Conforme a su etimología, la acción podría ser descrita por medio de otras palabras como adecuar o ajustar, hacer algo acorde con algo que tiene la misma forma;

- La interpretación conforme será siempre con relación a algo, por lo tanto es un término de comparación.

- La interpretación conforme es la actividad que consiste en buscar explicaciones de varios textos, por lo menos de dos, que sean compatibles entre sí.

- Siendo una actividad se debe suponer que el momento inicial es una situación de inconformidad o, por lo menos, de potencial inconformidad

Como con este concepto de interpretación conforme se incluye a la interpretación que resuelve conflictos de especialidad y cronológicos, se corre el riesgo de confusión sobre si hablamos o no de la directiva constitucional de interpretación conforme surgida de la reforma del artículo primero constitucional, o la que se hacía con base en el 133 constitucional. Al respecto, cabe hacer la distinción entre la interpretación conforme como técnica interpretativa y como institución jurídica; es decir, la primera como directiva retórica de interpretación, 133 , y la segunda, $1^{\circ}$ como directiva jurídica de interpretación. Esta última, busca hacer ajuste entre normas pero hacia una dirección específica: el respeto de los derechos humanos, es decir, no es un ajuste cualquiera. La interpretación conforme que no sea sobre derechos humanos será retórica. La conjunción entre Constitución y tratados internacionales, representa una interpretación sistemática entre ellos y la aplicación de los principios de interdependencia e indivisibilidad de los derechos humanos, que siempre ha de estar presente.

\section{Tendencias de decisiones judiciales en torno a la interpretación conforme}

El siguiente punto lo desarrollamos a partir de observar algunas decisiones dadas por la Corte, en torno a lo que considera interpretación conforme. Lo que nos interesa identificar son los cambios en el criterio en torno a dos cuestiones: la relación del orden jurídico mexicano con la propia constitución política y los tratados internacionales y la relación de las normas constitucionales con los tratados internacionales. Debido a cuestiones de espacio, sólo aludiremos a algunos aspectos de estas decisiones, pero señalaremos su referencia a fin de que el lector interesado las localice completas 
En 1992 se dijo por la corte: " Leyes federales y tratados internacionales. Tienen la misma jerarquía normativa...el tratado internacional no puede ser criterio para determinar la constitucionalidad de una ley ni viceversa..."18

Conforme a esta decisión vemos que la corte coloca a los tratados en igualdad de jerarquía a las leyes federales y a los tratados, pero por debajo de la constitución

En 1999, la Corte dijo "los tratados internacionales se encuentran en un segundo plano inmediatamente debajo de la Ley Fundamental y por encima del derecho federal y el local"19. De acuerdo a esta decisión, los tratados siguen estando debajo de la constitución pero por encima de las leyes federales

Estas decisiones son importantes toda vez que marcaron la pauta en la consideración del derecho internacional como referente de interpretación conforme.

También en 1999 y con relación al artículo 133 constitucional, los tribunales marcaron la pauta en lo que al llamado "control difuso de la constitución" se refiere cuando en dos jurisprudencias afirmaron "Control judicial de la constitución. Es atribución exclusiva del poder judicial de la federación" ${ }^{20}$ y la otra "Control difuso de la constitucionalidad de normas generales. No lo autoriza el artículo 133 de la constitución... el artículo 133 constitucional no es fuente de facultades de control constitucional para los Jueces del orden común..." ${ }^{21}$ Podemos comentar que conforme a estas anteriores decisiones el control de qué era o no constitucional se centraba en el poder judicial, federal, aun cuando, del propio artículo 133 constitucional, se facultaba a cualquier autoridad aplicar la constitución federal.

No fue sino hasta el 2011 y a partir de la condena de la Corte Interamericana de Derechos Humanos en contra de México en el Caso Rosendo Radilla, cuando los tribunales deciden lo siguiente: "De conformidad con lo previsto en el artículo 10. de la CPEUM, todas las autoridades del país, dentro del ámbito de sus competencias, se encuentran obligadas a velar no sólo por los derechos humanos contenidos en la Constitución Federal, sino también por aquellos contenidos en los instrumentos

18 Tesis Aislada P. C/92. Registro: 205596

19 Tesis: P. LXXVII/99. Registro: 192867

20 Jurisprudencia P./J. 73/99, Registro IUS 193558

21 Jurisprudencia P./J. 74/99. Registro No. 193435 
internacionales celebrados por el Estado Mexicano, adoptando la interpretación más favorable al derecho" 22 y posteriormente en 2012 se dicta la siguiente tesis "...las normas relativas a derechos humanos se interpretarán conforme a la Constitución y a los tratados internacionales en la materia, favoreciendo en todo tiempo a las personas la protección más amplia, ... y que la interpretación de aquélla y de las disposiciones de derechos humanos contenidas en instrumentos internacionales y en las leyes, siempre debe ser en las mejores condiciones para las personas [...]." ${ }^{23}$ En esta última decisión se puede observar como el principio de interpretación conforme ha de ir acompañado por el de pro persona, y está subordinado al mismo.

\section{Conclusiones}

De las decisiones aquí presentadas, se puede observar que se dieron cambios en las tendencias acerca de qué considerar por interpretación conforme así como de quién era la responsabilidad de establecerla ( jueces federales o locales o cualquier autoridad).Sin duda atrás de estas tendencias estuvieron presentes algunos factores externos a la toma de decisión, tales como factores políticos, sociales o económicos, mismos que no son evidentes en la decisión misma pero que pueden dar lugar a continuar la presente investigación.

Lo que nos parece más evidente a partir del cambio del artículo primero constitucional, es la responsabilidad de los jueces justificar sus decisiones, que siempre la han tenido, sin embargo en un estado de derecho constitucional, esta justificación ya tiene un marco a su medida: la defensa de los derechos humanos a favor de las personas.

22 Tesis Aislada: P. LXVII/2011(9a.). Registro: 160589

${ }^{23}$ Tesis 1a.XVIII/2012. Reg. IUS 160073 


\section{Bibliografía y Webgrafía}

ATIENZA, Manuel, Cuestiones Judiciales, ed. Fontamara, colección BEFDP No 91, México, 2001

CARBONELL, Miguel, Neo constitucionalismo y derechos fundamentales, Colombia, ed. Cevallos, 2011

CARBONELL, Miguel, Constitución, reforma constitucional y fuentes de derecho en México, $6^{\mathrm{a}}$ ed., México, Porrúa-UNAM, 2008

FERRAJOLI, Luigi, Democracia constitucional y derechos fundamentales. La rigidez de la Constitución $\quad y \quad$ sus garantías, http://www.fcje.org.es/wpcontent/uploads/file/jornada3/3_FERRAJOLlespa\%F1ol.pdf.

FERRAJOLI, Luigi, La teoría del derecho en el paradigma constitucional, México, Fontamara, 2010

GUASTINI, Riccardo, Estudios sobre la Interpretación Jurídica, Porrúa, México, 2001

KELSEN, Hans, Teoría Pura del Derecho, Ed. Porrúa, México, 1997

MORENO Rodríguez, Rodrigo, La administración pública federal en México, Universidad Nacional Autónoma de México, México, 1980

NINO Carlos S. Introducción al Análisis del Derecho, Ed. Ariel, Barcelona, 1987.

RAZ, Joseph, La autoridad del derecho. Ensayos sobre derecho y moral, Universidad Nacional Autónoma de México, México, 1985,

ZAGREBELSKY, Gustavo, El derecho dúctil. Ley derecho y justicia, Ed. Trotta, Madrid, 2011. 